\title{
Development of multichannel quartz crystal microbalances for MIP-based biosensing
}

\author{
Dieter Croux ${ }^{*, 1}$, Ans Weustenraed ${ }^{2}$, Paulius Pobedinskas' ${ }^{1}$ Frederik Horemans ${ }^{2}$, Hanne Diliën², \\ Ken Haenen ${ }^{1,3}$, Thomas Cleij ${ }^{2}$, Patrick Wagner ${ }^{1,3}$, Ronald Thoelen ${ }^{4}$, and Ward De Ceuninck ${ }^{1,3}$ \\ ${ }^{1}$ Institute for Materials Research (IMO), Hasselt University, Wetenschapspark 1, 3590 Diepenbeek, Belgium \\ ${ }^{2}$ Institute for Materials Research (IMO), Hasselt University, Agoralaan, Building D, 3590 Diepenbeek, Belgium \\ ${ }^{3}$ IMEC vzw, IMOMEC, Wetenschapspark 1, 3590 Diepenbeek, Belgium \\ ${ }^{4}$ XIOS University College, Agoralaan, Building H, 3590 Diepenbeek, Belgium
}

Received 12 October 2011, revised 14 December 2011, accepted 10 January 2012

Published online 6 April 2012

Keywords biosensor, finite element analysis, molecular imprinting, quartz crystal microbalance

*Corresponding author: e-mail dieter.croux@uhasselt.be, Phone: +32-11-268819, Fax: +32-11-268899

Molecularly imprinted polymers (MIPs) offer a huge potential in the development of cheap small-scale disposable biomimetic sensors. They are suited for a wide variety of biological targets and are compatible with many different measurement techniques such as gravimetric sensing and impedance spectroscopy. One potential sensor platform for MIP-based biosensors is the quartz crystal microbalance (QCM). A 4-channel MIP/nonimprinted polymer (NIP) coated QCM biosensor array was developed on a single piece of quartz crystal. To study cross- channel frequency coupling of the resonance modes, a simulation of crystal designs using finite element analysis (FEA) modeling was created. Based on these simulations and using reactive ion etching (RIE) to create mesa-like structures on the crystal surface, crosstalk can be severely reduced. The improved functionality compared to the traditional QCMs was demonstrated by employing these mesa-type multichannel QCM (MQCM) crystals as an L-nicotine biosensor platform.
1 Introduction The easy and accurate detection of specific molecules, present at very low concentrations, has always been a topic of significant scientific interest. It would allow better early stage monitoring of certain health conditions by detecting biomedically relevant molecules in patient samples. One promising line of research is molecular imprinting [1]. Molecularly imprinted polymers (MIPs) are synthetic biomimetic polymers with imprinted nanocavities that form preferential binding sites for the desired target molecule. MIPs have already been proven to be extremely suited for the detection of various low molecular weight targets such as histamine [2, 3], L-nicotine [4], and adenine [5]. As an added bonus, they are robust and completely inert over a wide range of different environments, with, for example, a wide temperature and $\mathrm{pH}$ range [6].

Two major sensor platforms are traditionally used in MIP-based biosensors. The first is based on electrochemical impedance spectroscopy $[7,8]$ and can detect target molecules in the low nanomolar range. The second is a gravimetric sensor platform based on the quartz crystal microbalance (QCM) [2, 4], which can detect targets from the high nanomolar up to the micromolar range. (c) 2012 WILEY-VCH Verlag GmbH \& Co. KGaA, Weinheim

For each MIP-sensor however, also a complementary non-imprinted polymer (NIP) reference is required. Synthesized identically as the MIP but without the template molecules, this polymer lacks the imprinted nanocavities. By measuring differentially between both MIP and NIP samples, actual target detection can be distinguished from environmental noise such as temperature fluctuations and non-specific binding events. Using a QCM sensor platform, a single functional biosensor would require at least two separate quartz crystals. This leads to larger sensor size, increased price tag, and further measurement errors, as each crystal is non-identical to the others due to imperfect fabrication techniques. While these crystal-induced errors are tiny compared to the thickness variation of the deposited MIP and NIP layers, the elimination of these errors would allow more accurate future research into improving the uniformity of the MIP deposition itself.

The solution to this problem is the development of a multichannel QCM (MQCM). By combining four separate electrodes on a single piece of AT-cut quartz, two MIP/NIP pairs can be measured independently with high accuracy. Recently, such a MQCM has already been successfully 
tested as a gas sensor [9] and similar designs have, for example, been used in the detection of red blood cells [10] and yeast cells [11]. Now a first attempt is made to develop them as a suitable transducer for a MIP-based biosensor. To examine frequency coupling and thus crosstalk between these four channels, finite element analysis (FEA) models were created of the proposed crystal design. Based on the results of these models, a reactive ion etching (RIE) processing step was added to create mesa-like structures on the crystal surface, severely lowering frequency coupling.

Finally, for the first time a MIP sensor array for L-nicotine was fabricated using these 4-channel MQCM crystals and successfully tested as a compact biosensor platform.

\section{Experimental}

2.1 MQCM biosensor basics A QCM consists of a thin wafer of AT-cut quartz with electrodes patterned on both surfaces. When excited, the piezoelectric properties of the quartz crystal induce a thickness shear mode (TSM) resonance vibration. The resonance frequency is inversely dependent upon the crystal thickness and established by fulfilling a standing transverse acoustic wave condition:

$$
f_{0}=\frac{\sqrt{\mu_{\mathrm{q}} / \rho_{\mathrm{q}}}}{2 t_{\mathrm{s}}},
$$

where $\mu_{\mathrm{q}}$ and $\rho_{\mathrm{q}}$ are, respectively, the shear modulus and the density of quartz and $t_{\mathrm{s}}$ is the crystal thickness.

This thickness of the vibrating membrane is increased by the deposition of the electrodes, the bio-mimetic layer and the binding of the target molecules. Thus, changes in the mass loading of the QCM are translated into inverse changes in the resonance frequency. The resulting frequency shift $\Delta f$ due to a mass deposition $\Delta m$ on the piezoelectrically active surface area $A_{\text {piezo }}$ is described by the Sauerbrey equation [12], assuming more or less rigid adherence of the mass deposition layer to the crystal surface:

$$
\Delta f=-\frac{2 f^{2} \Delta m}{A_{\text {piezo } \sqrt{\mu_{\mathrm{q}} \rho_{\mathrm{q}}}}} .
$$

Operating in a liquid environment, the resonance frequency also depends upon the viscosity $\eta_{\mathrm{L}}$ and the density $\rho_{\mathrm{L}}$ of the liquid [13]:

$$
\Delta f=-f_{0}^{2 / 3} \sqrt{\frac{\eta_{\mathrm{L}} \rho_{\mathrm{L}}}{\pi \mu_{\mathrm{q}} \rho_{\mathrm{q}}}} .
$$

Combining (2) and (3) will give a rough estimation of the behavior of a mass-loaded QCM in a liquid environment. A more accurate estimation would need to take into account the various reflections of the vibration waveform caused by, for example, the crystal sample holder and the liquid container. Figure 1 shows the equivalent circuit of this mass-loaded, liquid-loaded QCM [14], based on the traditional Butterworth-van-Dyke equivalent circuit. The quartz crystal itself

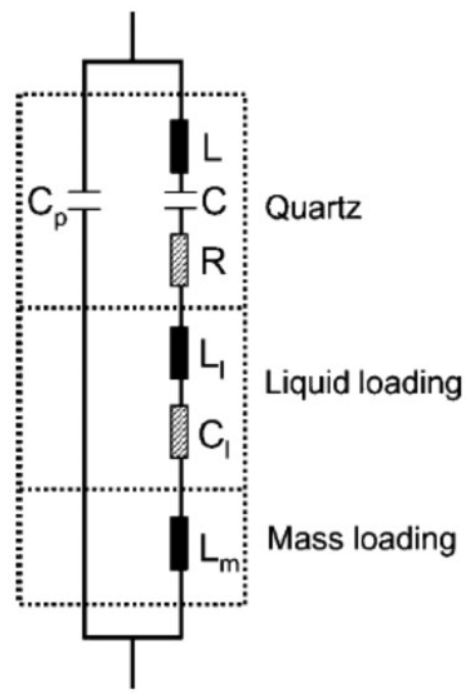

Figure 1 Equivalent circuit of a coated QCM in liquid [14].

contains an inductance $L$ for the energy storage during vibration, a serial capacitance $C$ as a measure of mechanical elasticity, a parallel capacitance $C_{\mathrm{p}}$ for the dielectric quartz substrate between the electrodes and a resistance $R$ for all miscellaneous energy losses. Added to these are an inductance $L_{1}$ from the vibration of the liquid and a conductance $C_{1}$ from the losses caused by the viscosity of the liquid. In case of a thin rigid layer, the mass loading is represented by an inductance $L_{\mathrm{m}}$.

Not only do these additional parameters shift the resonance frequency, they also dampen and broaden the measured resonance peak. The $Q$-factor is defined as the ratio between stored energy and dissipated energy of a quartz resonator. It is a dimensionless measure of the quality of the resonator and can be calculated for an unloaded QCM in air as:

$$
Q=\frac{\omega L}{R}=\frac{1}{\omega R C} .
$$

It is intuitive that the addition of mass loading and liquid loading parameters in Eq. (4) will severely lower the $Q$-factor of a MIP-based QCM biosensor [15].

It has been found that most of the vibration energy of a QCM can be trapped within the region between the electrodes [16]. This makes it possible to construct a highquality, high-density MQCM crystal. However, this energy trapping mechanism is not perfect and adding more than one electrode pair in close proximity to each other on a quartz crystal always introduces a certain amount of frequency coupling between the channels. The main TSM vibration couples with other vibrational modes such as thickness flexural mode (TFM), face shear mode (FSM) and other spurious modes. By far the largest coupling occurs between TSM and TFM due to their similar displacement field, as described by Mindlin and Lee [17]. 
A recent development to increase energy trapping in the electrode region is the introduction of mesa-shaped quartz structures underneath the electrodes [18-21]. If the quartz thickness in the excited region between the electrode pairs is thicker than the surrounding quartz, the resonance frequency of that channel would no longer be identical to the resonance frequency of the bulk quartz and the vibration energy stays better confined in the electrode region. This would allow much closer electrode distribution while minimizing crosstalk.

In addition, mass absorption on the electrode surface has an added benefit of moderately reducing the strength of frequency coupling by dampening the vibration energy and detuning the two resonators [22].

\subsection{Finite element analysis modeling of a} MQCM The FEA method is a very powerful numerical technique for the accurate modeling of complex structures and optimization of design parameters. To study the influence of frequency coupling on the proposed crystal design, a simplified model of a piece of AT-cut quartz crystal patterned on each side with four aluminum electrodes, both with and without mesa structures, was composed. The electrodes are patterned into four pairs of overlapping electrodes, creating four different channels on the MQCM. The model, created using the COMSOL software package, is based on a piezoelectric eigenfrequency calculation with added damping, dielectric loss and coupling loss parameters, coupled to an isotropic linear elastic material model for the electrodes. The mesh can be seen in Fig. 2, while its dimensions and properties are listed in Table 1 . The number of elements in the mesh had to be kept rather low so the model could be calculated in a reasonable time period. This unfortunately introduces a certain inevitable amount of noise into the resulting eigenfrequencies. These simulations were primarily intended as a design aid and quick testbed for new ideas, and not as an in-depth and highly accurate study.

The base crystal is a standard $1 \mathrm{in}$. AT-cut quartz disc with a resonance frequency expected around $5.03 \mathrm{MHz}$. Electrodes should be round to prevent noise from being generated by the discontinuities in the acoustic wave caused by, for example, the corners of rectangular electrodes [15].

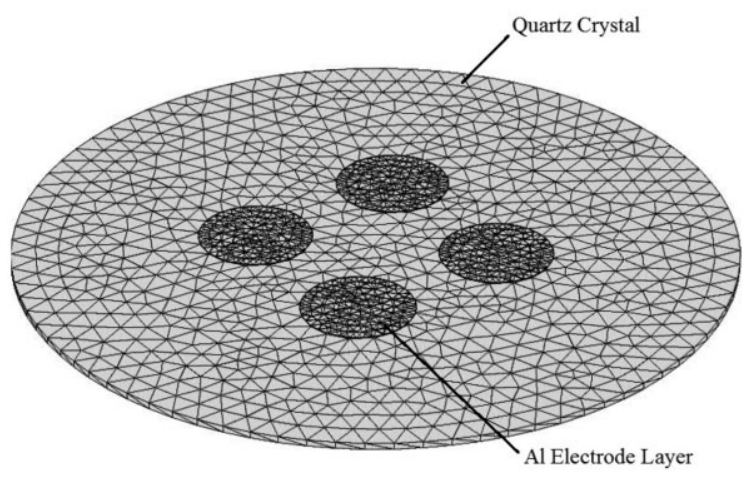

Figure 2 Finite element model of a 4-channel MQCM crystal.
Table 1 Dimensions of the quartz crystal microbalance and properties of the electrodes and mesh.

value

dimension

thickness of quartz $(\mu \mathrm{m})$

333

diameter of quartz $(\mathrm{mm})$

25.4

thickness of electrodes $(\mathrm{nm})$

100

diameter of electrodes $(\mathrm{mm})$

4

distance between two adjacent electrodes $(\mathrm{mm})$

1

electrode properties

density of $\mathrm{Al}\left(\mathrm{kg} / \mathrm{m}^{3}\right)$

elastic modulus $\left(\times 10^{9} \mathrm{~N} / \mathrm{m}^{2}\right)$

2700

70

Poisson's ratio

mesh properties

total number of elements

26543

number of elements covering thickness quartz

number of elements covering thickness electrode

number of elements covering thickness mesa

8

1

10

Also, the electrode diameter and height were designed to be large enough to cause a clean fundamental TSM and prevent coupling with odd higher overtones of surface shear mode in a traditional QCM [23]. Inter-electrode distance is relatively small to keep array size as compact as possible and make frequency coupling between two or more electrode regions a major influence in resonance behavior. Aluminum was chosen as electrode material due to its inherent thin oxide surface layer, which is required for the growth of the MIP and NIP coating.

Lu et al. [24] and Shen et al. [25] already demonstrated the existence of frequency interference between symmetrical channels of a 2-channel planar MQCM. In theirFEA model, when the resonance frequencies of bothchannnels are close to each other and both are excited simultaneously, the vibration shapes were shown to split into a parallel and an anti-parallel resonance due to lateral coupling between the channels. The frequency difference between these two modes, called the splitting frequency, was demonstrated to provide a convenient evaluation of the coupling strength. This splitting frequency decreased with increasing electrode thickness and inter-electrode spacing. Ideally, the splitting frequency would be 0 and each channel would behave as an independent quartz microbalance.

Calculating the eigenfrequencies of the 4-channel planar MQCM models during simultaneous excitation of all four electrodes, it is clear that this splitting phenomenon and thus frequency coupling occurs strongly between all four electrode regions. Figure 3 shows the vibration shapes of the four resonances of the TSM mode for a planar crystal. AT-cut quartz is anisotropic in the $X-Y$ plane, causing nonuniform wave propagation. The electrodes were positioned along these axes, which would maximize the frequency coupling effects according to $\mathrm{Lu}$ et al. [22]. The four resonances can be grouped accordingly, with one parallel mode, and three anti-parallel modes aligned along the $X, Y$, and $X Y$ axes of the crystal. Much energy gets trapped in the 


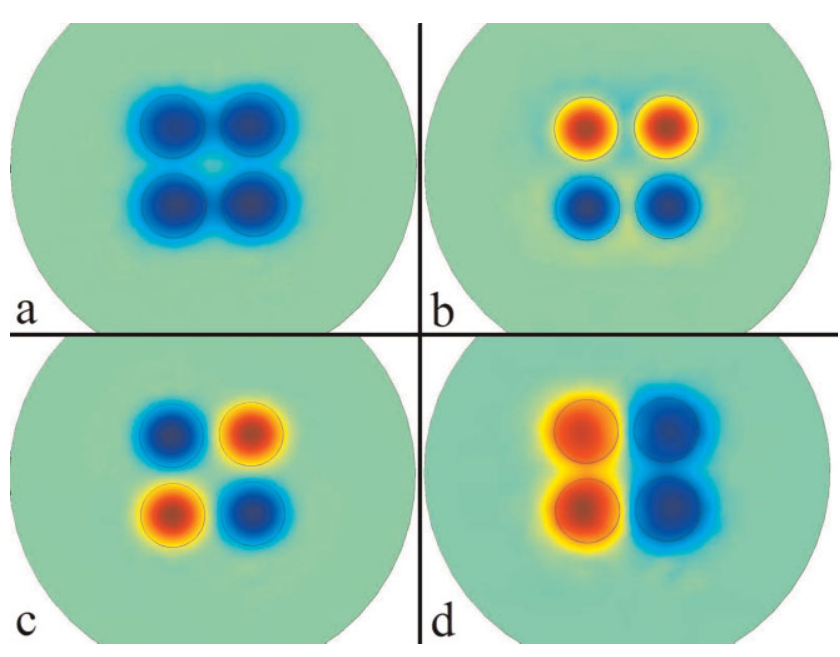

Figure 3 (online color at: www.pss-a.com) The four resonance vibration shapes of a planar 4-channel MQCM showing lateral coupling: parallel resonance (a), anti-parallel $X$ resonance (b), anti-parallel $X Y$ resonance (c), and anti-parallel $Y$ resonance (d).

quartz between two electrodes, most notably in the parallel and anti-parallel $Y$ modes. This can be seen from the displacement overlap, which strongly induces the frequency coupling. The splitting frequency $f_{\mathrm{s}}$ for a 4-channel MQCM will now be defined as:

$$
f_{\mathrm{s}}=f_{\text {res,max }}-f_{\text {res,min }},
$$

where $f_{\text {res,max }}$ and $f_{\text {res,min }}$ are, respectively, the maximum and minimum frequency where these four parallel and antiparallel resonances occur.

As mentioned earlier, the addition of the mesa structures increases energy trapping in the electrode region. As a result, frequency coupling reduces. Mesa structures were included in the FEA model by keeping the quartz thickness underneath the electrodes constant at $333 \mu \mathrm{m}$ and thinning the rest

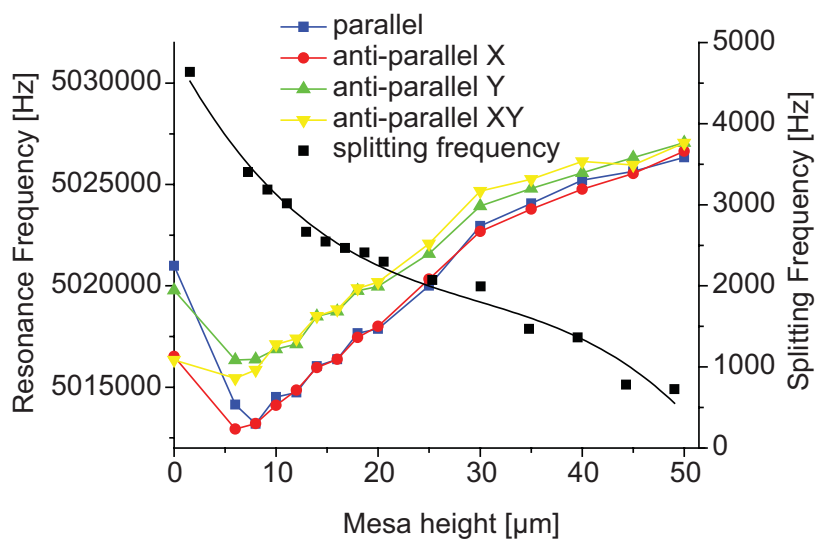

Figure 4 (online color at: www.pss-a.com) Evolution of the resonance frequencies and splitting frequency with increasing mesa height.

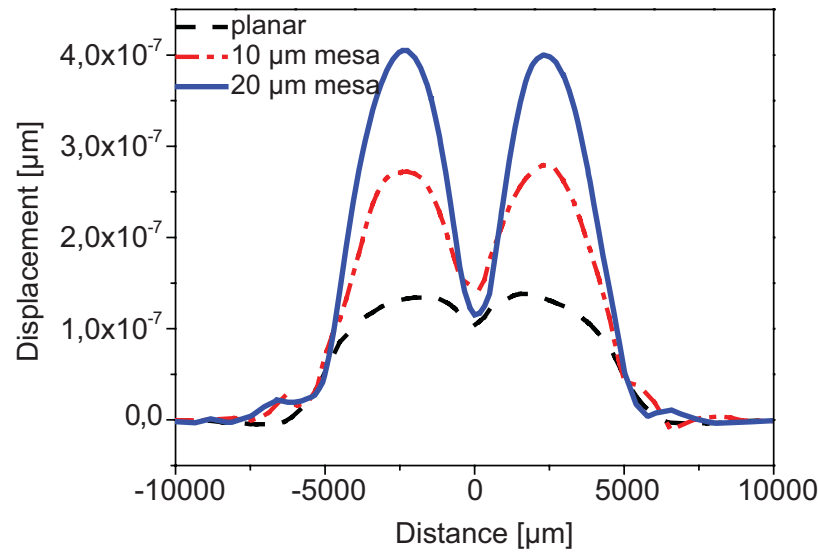

Figure 5 (online color at: www.pss-a.com) Amplitude of the parallel resonance displacement across two adjacent electrodes for different mesa height.

of the quartz accordingly. A simulation of the evolution of the resonance frequencies and corresponding decrease in splitting frequency for increasing mesa height can be seen in Fig. 4. It is clear that even despite large mesa height $(50 \mu \mathrm{m})$, the splitting frequency never reached 0 and thus frequency coupling would not entirely be eliminated. It also seems that the resonance frequency slowly increases with increasing mesa height, while the initial introduction of mesa structures causes a relatively large drop in resonance frequency. The exact cause of this is unknown, but likely related to the other frequency coupling phenomena. Also noticeable is how, respectively, the parallel and anti-parallel $X$ and the antiparallel $Y$ and anti-parallel $X Y$ resonance frequencies are grouped closely together.

The improved energy trapping effect of the mesa structures can be seen in Fig. 5, which shows the simulated amplitude of the parallel resonance displacement for both a planar, a $10 \mu \mathrm{m}$ mesa and a $20 \mu \mathrm{m}$ mesa 4-channel MQCM, as seen along two adjacent electrodes. The electrodes, $4 \mathrm{~mm}$ in diameter, are each centered at $2500 \mu \mathrm{m}$ from the crystal center. Increased mesa height clearly offers a better vibration shape, higher displacement during resonance, and relatively less vibration energy trapped in the area between both electrode pairs $(-500 \mu \mathrm{m}<x<500 \mu \mathrm{m})$.

\subsection{Fabrication of the L-nicotine sensor With} these FEA models and simulations, the viability of the proposed 4-channel MQCM crystal design with mesas has been demonstrated. Based on the conclusions from these models, a fully functional nicotine MIP biosensor was fabricated. A schematic overview of the fabrication process is given in Fig. 6.

The RIE of the mesa structures in the quartz crystal was performed in a $\mathrm{CF}_{4} / \mathrm{O}_{2}$ gas discharge plasma (10 Pa pressure). The top side of the quartz crystal, covered with PDMS as a protective mask, was placed on a molybdenum stage, which was biased with DC-pulses. The power of the DC-pulses was $300 \mathrm{~W}$. The flow rate of tetrafluoromethane 


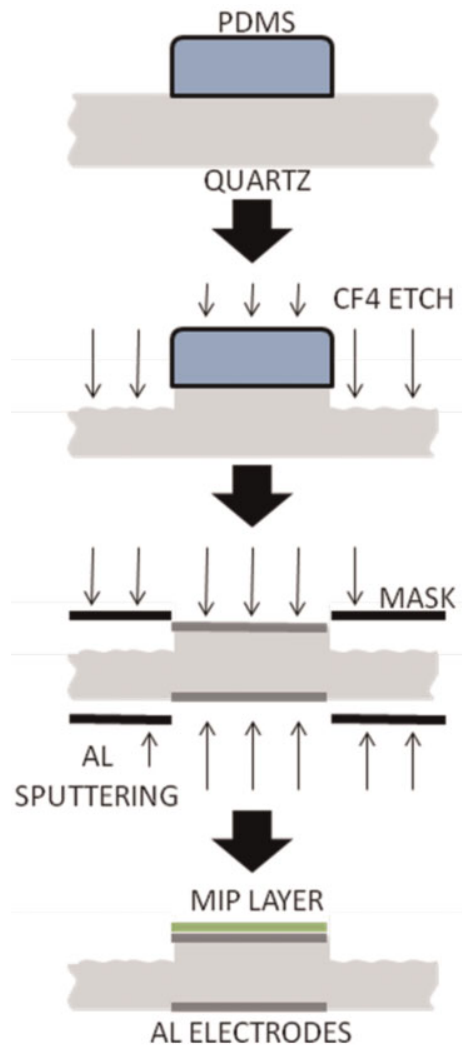

Figure 6 (online color at: www.pss-a.com) Schematic overview of the manufacturing steps.

and oxygen into the chamber consisted of 42 and $6 \mathrm{sccm}$, respectively. Given these settings, an etch rate of around $88 \mathrm{~nm} / \mathrm{min}$ and a final mesa height of $8 \pm 0.05 \mu \mathrm{m}$, as measured by a Dektak profilometer with $5 \AA$ vertical resolution, was achieved. Deeper etching provided more irregular results and started noticeably underetching the PDMS mask. No further polishing of the etched areas was performed, as these regions are not of interest for the functionality of the sensor.

After etching, the PDMS mask was removed and replaced by a mechanical mask to sputter the $\mathrm{Al}$ electrodes on both sides of the crystal. These were deposited by DC-pulsed magnetron sputtering. The sputtering target, $\sim 4$ in. aluminum (99.9995\%), was held on the water-cooled magnetron cathode.

As an optional first step, a $10 \mathrm{~nm}$ titanium layer could be sputtered from a titanium $(99.995 \%)$ target to increase electrode adhesion to the crystal, thus improving the resistance of the electrodes to mechanical damage. The distance between the target and the copper sample holder was $12 \mathrm{~cm}$. The base pressure in the sputtering chamber was below $5 \times 10^{-4} \mathrm{~Pa}$. The $\mathrm{Al}$ layers were sputtered in argon gas discharge plasma ( $\sim 0.3 \mathrm{~Pa}$ pressure) with a thickness of $100-200 \mathrm{~nm}$. The argon flow rate of $50 \mathrm{sccm}$ was regulated by a mass flow controller. Also, the samples were not additionally heated during the deposition process.
For the preparation of the biomimetic MIP layer, this thin film of polymer with selective binding sites for L-nicotine needed to be anchored to the MQCM crystal surface. Ethylene glycol dimethacrylate (EGDM), methacrylic acid (MAA), 3-methacryloxypropyltrimethoxy-silane (y-MPS), and the target molecule L-nicotine were purchased from Acros. Prior to polymerization, the stabilizers in the MAA and EGDM were removed by filtration over alumina. Azobisisobutyronitrile (AIBN) was purchased from Fluka. All solvents were of analytical grade and purchased from Acros and used without further purification.

The samples were first cleaned by mild ultrasonication in isopropanol for $2 \mathrm{~min}$. The cleaned surface was then subjected to a solution of $10 \%$ y-MPS in toluene for $3 \mathrm{~h}$ under nitrogen atmosphere. Afterwards the crystals were rinsed with toluene and ethanol for $5 \mathrm{~min}$ to remove the unbound silane moieties. For the preparation of the MIP itself, a mixture of MAA (12.5 mmol), EGDM (25.2 mmol), and AIBN (1.5 mmol) was dissolved in $7 \mathrm{ml}$ toluene together with the template molecule L-nicotine $(6.41 \mathrm{mmol})$. To exclude oxygen in the mixture, it was degassed for $10 \mathrm{~min}$ with $\mathrm{N}_{2}$. By using a PDMS mold to keep the MIP localized on top of the electrodes, this mixture was introduced onto the MQCM surface using a PDMs mold, where it was heated at $65^{\circ} \mathrm{C}$ for $10 \mathrm{~min}$ to induce the polymerization. After polymerization the solid MIP was extensively washed using repeated flushings with ethanol. The 4-channel MQCM crystals were covered with two MIP layers and two NIP layers synthesized in the same way but without the presence of the target molecule.

The finalized nicotine sensor crystal is shown in Fig. 7. On top of the crystal, a PDMS microchamber was attached to serve as miniature flowcell and physical protection of the MIP/NIP layers. A FEA model of the flow inside this microchamber, using a k- $\varepsilon$ model for Navier-Stokes turbulent flow with a fixed inflow speed, was created to guarantee the absence of localized concentration gradients caused by flow turbulence. The results of this simulation can be seen in Fig. 8.

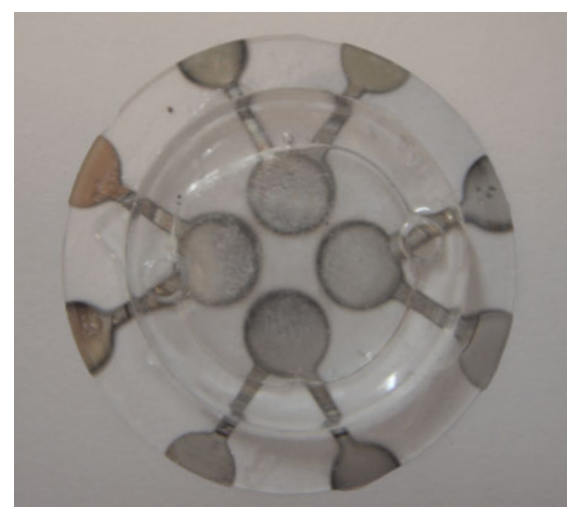

Figure 7 (online color at: www.pss-a.com) The final 4-channel MQCM crystal with MIP/NIP layer grown on the center electrodes and a PDMS microchamber. 


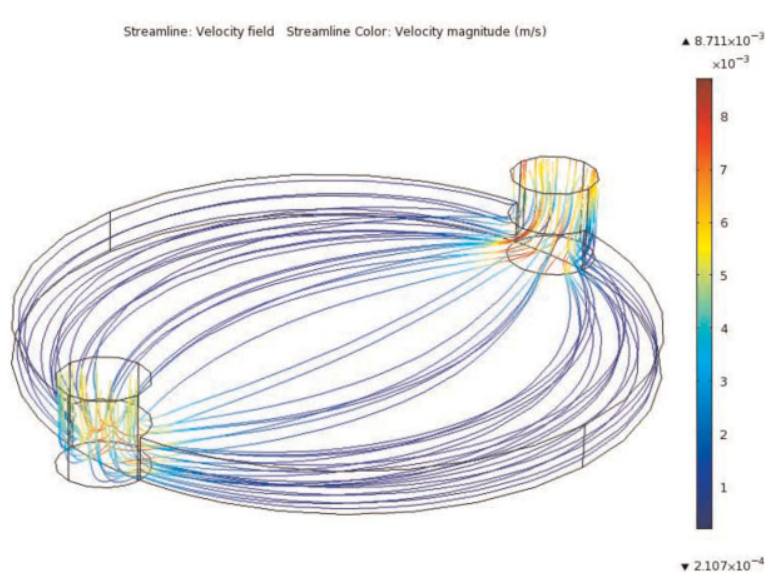

Figure 8 (online color at: www.pss-a.com) FEA simulation of the flow inside the PDMS microchamber, showing the complete absence of turbulence.

Finally, the measurement setup for these L-nicotine MQCM biosensors is shown in Fig. 9. The PDMS microchamber is connected to a peristaltic pump which continually flows the nicotine target in a buffer solution across the top electrodes of the crystal. A small multiplexer PCB connects these electrodes with a HP4194A Impedance Analyzer, which is used to perform frequency sweeps to determine the resonance frequency of the selected channel. The entire setup is controlled by a home-made program in NI LabVIEW, which will also translate the frequency sweep data from the impedance analyzer into time-based resonance frequency shifts.

\section{Results and discussion}

3.1 Characterization of the MQCM First, characterization measurements were performed on the crystals. These experiments were done by measuring the admittance parameters of each individual channel in a span of

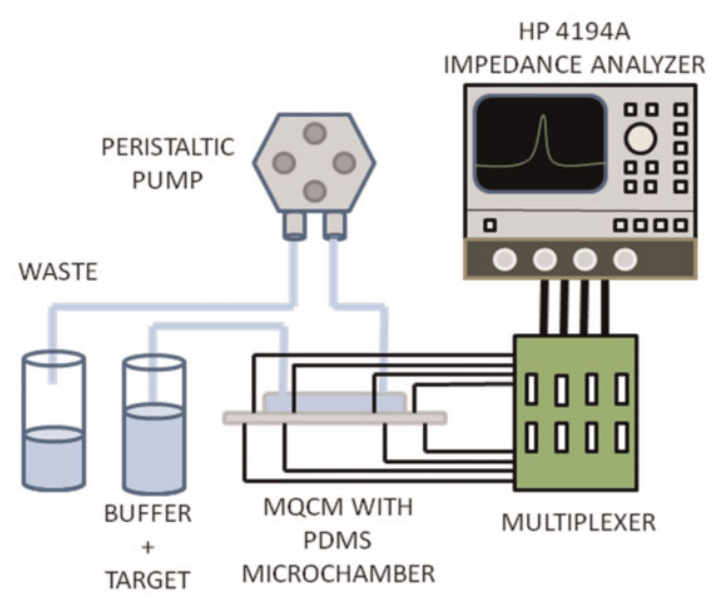

Figure 9 (online color at: www.pss-a.com) Schematic overview of the measurement setup. Not shown is the laptop used as controller and data processor.

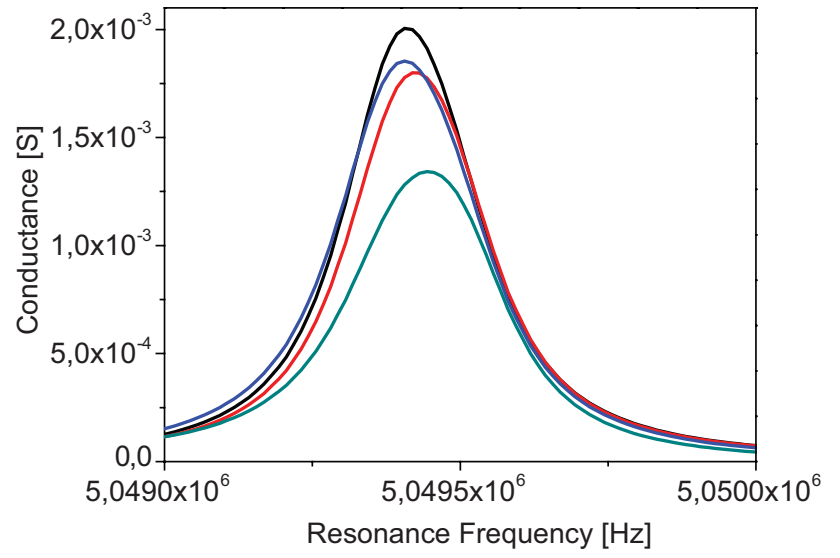

Figure 10 (online color at: www.pss-a.com) The conductance peaks at resonance frequencies of the four channels of an $8 \mu \mathrm{m}$ mesa MQCM.

several $\mathrm{kHz}$ around its resonance frequency. To further decrease the effects of frequency coupling, only one single electrode pair was excited at the same time. This provided increased measurement accuracy at the cost of being four times slower to measure all four channels.

Figure 10 shows the conductance of the four resonance peaks of an $8 \mu \mathrm{m}$ mesa MQCM before the addition of the biomimetic layer. There was some variation in peak height, but the resonance frequencies appeared much closer together than predicted from the FEA model, most likely due to the lack of simultaneous excitation of all four electrode pairs. The peaks were still grouped together in pairs, with approximately $100 \mathrm{~Hz}$ difference, depending on the individual sample, between both pairs. This would indicate only minimal crosstalk between the four channels even before the mass loading from the final fabrication step detuned them further. $Q$ factors were found to be between 12000 and 15000 , depending on the sample. In comparison, the $Q$-factor for commercially available 1 in. single channel QCMs tends to be between 15000 and 20000 .

The four peaks are non-identical due to the different locations on the quartz and the slight variation in electrode thickness from the $\mathrm{Al}$ sputtering. Based on these results, the MQCM crystal with $8 \mu \mathrm{m}$ mesas was expected to be highly suitable as a sensor platform for any type of gravimetric sensor application, including the MIP-based nicotine biosensor.

The effect of the mesa structure energy trapping and the influence of mass loading and liquid loading on the crystal resonance is demonstrated in Fig. 11. For this particular sample, the fabrication process was slightly modified so that the electrodes were patterned first and only-afterwards the mesas were etched. This gave a better opportunity to track the evolution of the resonance frequency for each step. The resonance peaks from the planar MQCM were all between 0.8 and $1 \mathrm{mS}$ high, with the four resonance frequencies laying inside a $0.9 \mathrm{kHz}$ span. The introduction of a mesa underneath the electrode gave a much higher peak in 


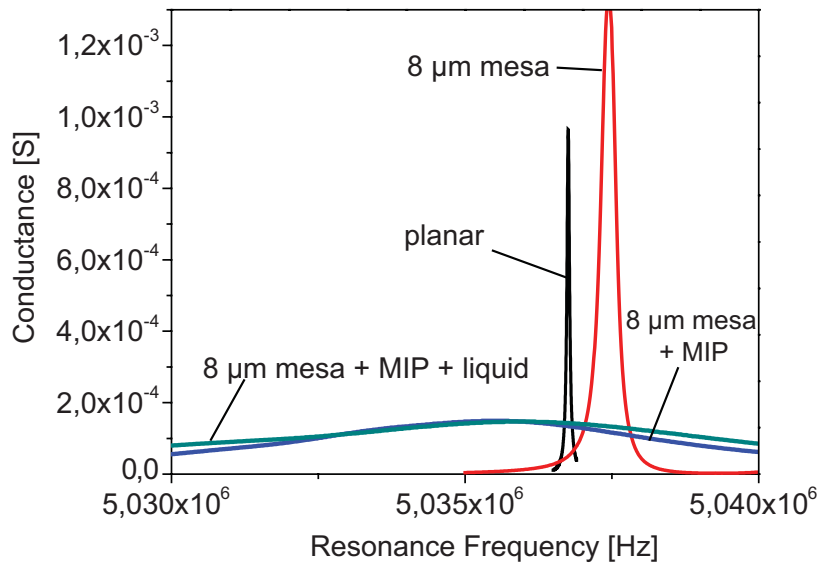

Figure 11 (online color at: www.pss-a.com) Evolution of the resonance peak from one of the channels after each step in the MQCM fabrication process.

conductance at a slightly higher resonance frequency, as predicted by the FEA model.

Adding the MIP coating to the top electrode caused a severe damping of the vibration and a small frequency shift of a few kilohertz downwards due to the additional mass loading. The resonance peak was now seven times smaller and more than ten times wider than before, making it much more susceptible to measurement noise. Adding liquid loading was a relatively minor additional damping factor compared to the mass loading caused by the MIP layer.

3.2 MQCM as L-nicotine sensor To test the finalized MQCM MIP biosensor, several samples were subjected to varying low concentrations of a L-nicotine solution in an PBS buffer of $\mathrm{pH}$ 7. Starting with only pure PBS in the PDMS flowchamber, every $30 \mathrm{~min}$ a new L-nicotine concentration was introduced to the sensor. At such low concentrations, an equilibrium state would definitely require $20 \mathrm{~min}$ to be reached at the very least. Admittance parameter frequency sweeps in a span of a few kilohertz around each channel's

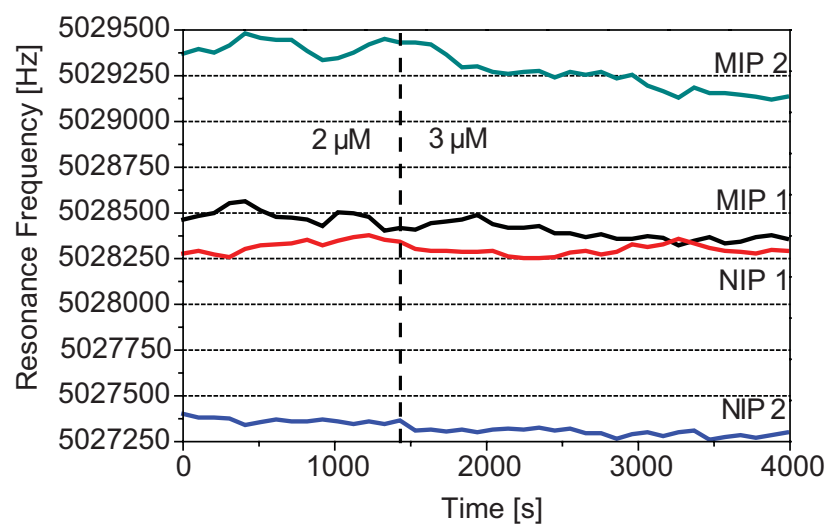

Figure 12 (online color at: www.pss-a.com) Example of the reaction of the four channels of a MQCM L-nicotine sensor to a change in L-nicotine concentration in an PBS buffer of $\mathrm{pH} 7$. original resonance frequency were performed continuously throughout, from which the LabVIEW measurement software automatically processed the relevant frequency shifts. To reduce environmental and mechanical reflection noise, each data point is the averaged result of four frequency sweeps.

Figure 12 shows an example of the typical reaction of the $2 \mathrm{MIP} / \mathrm{NIP}$ pairs to a slight increase in L-nicotine concentration. Each such detection step is processed by the software as a separate measurement. It can be noted that both MIPs reacted differently to this change. The cause of this could be found in the quality of the MIP layer deposition. The MIP2 layer was slightly thicker and more planar than MIP1. As a result, the MIP2 channel offered more binding spots for the L-nicotine molecules and thus provided better sensitivity at the trade-off of a longer time required to reach equilibrium. The deposition of more or less identical MIP/NIP layers remains challenging. The NIP2 channel also experienced a slight downward shift in resonance frequency, most likely due to crosstalk from the adjacent MIP2 channel. A certain amount of noise can still be seen in the measurement data and is unfortunately unavoidable given the physical behavior of a quartz resonator.

The end result of many such concentration change measurements on a second MQCM L-nicotine biosensor can be seen in Fig. 13. These curves were constructed by simply averaging the differential time-based data (such as shown in Fig. 12) for each concentration step. The frequency shifts from both MIP/NIP pairs are plotted differentially to eliminate the temperature noise and non-specific binding events. The resulting concentration curves show the expected slight logarithmic tendencies that denote the beginning saturation of the MIP binding sites. Once again the MIP channels differ in sensitivity due to the non-identical layer deposition. Full saturation, and thus the detection limit, is expected around $15 \mu \mathrm{M}$, again dependent on the quality of the MIP deposition.

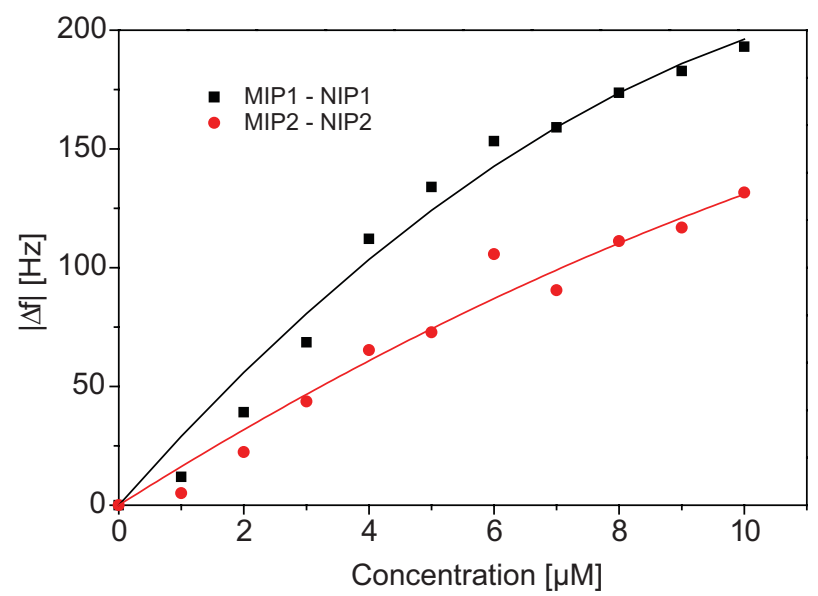

Figure 13 (online color at: www.pss-a.com) Concentration curves for two nicotine MIP/NIP pairs obtained from a MQCM with $8 \mu \mathrm{m}$ mesa structures. 
4 Conclusion The 4-channel MQCM has been demonstrated to be a suitable miniature sensor platform for MIPbased biosensors. Coated by biomimetic MIP and reference NIP layers, relatively low concentrations of L-nicotine could be detected. This coating layer and the surrounding presence of a liquid on the sensor surface unfortunately severely degraded the performance of the resonator. Low liquid viscosity and an extremely thin biomimetic layer are strongly recommended for optimal performance.

While relatively easy and cost-efficient to fabricate these biosensors, care has to be taken to prevent crosstalk by frequency coupling between the channels. In this regard, FEA proved a very suitable and convenient tool in the search for improved crystal designs. With the introduction of mesalike structures on the crystal surface, frequency coupling effects could already be severely reduced. The additional mass and liquid loading present in the fully functionalized biosensor further minimize these effects. Additional development into even more efficient MQCM crystals using multiple etching steps is already ongoing.

One advantage of these design techniques for 4-channel MQCM crystals is that they could easily be expanded into larger sensor arrays of 8, 16, 32, 64, etc., channels. Ideally, as each individual electrode pair would operate as if it were a separate crystal, it matters not how many electrode pairs would be present on the quartz. In reality however, some minor crosstalk is likely to be unavoidable.

Acknowledgements We would like to thank the Special Research Fund (BOF) of Hasselt University to provide us with the necessary financial resources. Furthermore, we owe many thanks to the expertise and knowledge of Jan Mertens, Johan Soogen, Johnny Baccus, and Lieven De Winter for technical support. This work is supported by the "Life Science Initiative of the Province Limburg."

\section{References}

[1] C. Alexander, H. S. Andersson, L. I. Andersson, R. J. Ansell, N. Kirsch, I. A. Nicholls, J. O'Mahony, and M. J. Whitcombe, Mol. Recognit. 19, 106-180 (2006).

[2] F. Horemans, J. Alenus, E. Bongaers, A. Weustenraed, R. Thoelen, J. Duchateau, L. Lutsen, D. Vanderzande, P. Wagner, and T. J. Cleij, Sens. Actuators B 148, 392-398 (2010).

[3] E. Bongaers, J. Alenus, F. Horemans, A. Weustenraed, L. Lutsen, D. Vanderzande, T. J. Cleij, F. J. Troost, R.-J. Brummer, and P. Wagner, Phys. Status Solidi A 207, 837-843 (2010).
[4] R. Thoelen, R. Vansweevelt, J. Duchateau, F. Horemans, J. D'Haen, L. Lutsen, D. Vanderzande, M. Ameloot, M. vandeVen, T. J. Cleij, and P. Wagner, Biosens. Bioelectron. 23, 913-918 (2008).

[5] A. Pietrzyk, S. Suriyanarayanan, W. Kutner, R. Chitta, M. E. Zandler, and F. D'Souza, Biosens. Bioelectron. 25, 2522-2529 (2010).

[6] P. K. Owens and L. Karlsson, Trends Anal. Chem. 18, 146154 (1999).

[7] P. Cooreman, R. Thoelen, J. Manca, M. vandeVen, V. Vermeeren, L. Michiels, M. Ameloot, and P. Wagner, Biosens. Bioelectron. 20, 2151-2156 (2005).

[8] J. Broeders, S. Duchateau, B. van Grinsven, W. Vanaken, M. Peeters, R. Thoelen, T. J. Cleij, P. Wagner, and W. De Ceuninck, Phys. Status Solidi A 208, 1357-1363 (2011).

[9] X. Jin, Y. Huang, A. Mason, and X. Zeng, Anal. Chem. 81, 595-603 (2009).

[10] A. Seifner, P. Lieberzeit, C. Jungbauer, and F. L. Dickert, Anal. Chim. Acta 651, 215-219 (2009).

[11] F. Dickert, O. Hayden, P. Lieberzeit, C. Palfinger, D. Pickert, U. Wolff, and G. Scholl, Sens. Actuators B 95, 20-24 (2003).

[12] G. Sauerbrey, Z. Phys. 155, 206-222 (1959).

[13] K. K. Kanazawa and J. G. Gordon, III, Anal. Chim. Acta 175, 99-105 (1985).

[14] J. Auge, P. Hauptmann, J. Hartmann, S. Rösler, and R. Lucklum, Sens. Actuators B 24/25, 43-48 (1995).

[15] H. Anderson, M. Jönsson, L. Vestling, U. Lindberg, and T. Aastrup, Sens. Actuators B 123, 27-34 (2007).

[16] W. Shockley, D. R. Curran, and D. J. Koneval, J. Acoust. Soc. Am. 41, 981-993 (1967).

[17] R. D. Mindlin and P. C. Y. Lee, Int. J. Solids Struct. 2, 125 139 (1966).

[18] K. Hirama, Y. Aoyama, and M. Naito, Jpn. J. Appl. Phys. 36, 6432-6436 (1997).

[19] A. Ishizaki, H. Sekimoto, D. Tajima, and Y. Watanabe, Proceedings of the IEEE Ultrasonics Symposium, 913-916 (1995).

[20] H. Sekimoto, S. Goka, and Y. Watanabe, Proceedings of the 1998 IEEE International Frequency Control Symposium, 964-967 (1998).

[21] S. Goka, H. Sekimoto, and Y. Watanabe, Proceedings of the IEEE/EIA International Frequency Contr. Symposium, 397-400 (2000).

[22] F. Lu, H. P. Lee, P. Lu, and S. P. Lim, Sens. Actuators A 119, 90-99 (2005).

[23] H. Jiang, Proceedings of the Chinese Control and Decision Conference CCDC '09, 3618-3621 (2009).

[24] F. Lu, H. P. Lee, and S. P. Lim, IEEE Proc. Sensors 2, 888892 (2003).

[25] F. Shen, P. Lu, S. J. O'Shea, and K. H. Lee, Sens. Actuators A 111, 180-187 (2004). 